\title{
Knowledge based Jitter Analysis in Power System Control
}

RAMESH K. RAYUDU

\author{
SANDHYA SAMARASINGHE \\ Member \\ Dept. Natural Resources Engineering, \\ Lincoln University, \\ Canterbury, \\ New Zealand.
}

DON KULASIRI

\begin{abstract}
This paper presents the development of an expert system TPAISA $^{1}$ for fault analysis of analog problems in power system control. The objectives are to identify the problem analogs and to establish the fault diagnosis for each analog signal.

These objectives are accomplished by building the knowledge base which contains the heuristics of the expert and using it to identify the problem analogs and the equipment responsible for it. The knowledge base has been divided into three different modules; a blackboard architecture for efficient use of the modules is developed. The approach is validated by experimental results carried on the actual power system.
\end{abstract}

Keywords: Expert Systems, Fault Analysis, Fault Diagnosis, Power Systems, Analog Measurements, Knowledge Engineering.

\section{INTRODUCTION}

New Zealand's power system is controlled by two control centres, one in the South Island and the other one in the North Island, and cach centre is connected to a real-time computer network. Our research is concerned with the South Island power system which is controlled by South Island Control Centre (SICC). This control centre is equipped with a SCADA (Supervisory Control and Data Acquisition) system which provides the operators with the low level validation of the data by communicating with daia concentrators which control several Remote Terminal Units (RTUs) (Figure 1). In total, there are 7 data concentrators and 82 RTUs in South Island power system. Analogue, Control, and Status indication values from the power plants, switch yards, and sub stations are fed into RTUs which are sent to SCADA through data concentrators (Figure 1). Data concentrators manage the data sent by RTUs beforc sending it to the control centre. Research described here is related only to the analog values and not to the control and status values which indicate the status of the equipment. Analogue values are a measure of voltage or electrical current such as Power (MW), Voltage (V), Power Reactance (MV), and Current (A) are converted to digital signals by a transducer before sending to SCADA via Input/Output cards in the RTUs.

\footnotetext{
1 The funding from South Island Control Centre, Trans Power NZ Ltd., Christchurch, New Zealand, for the project is greatly appreciated.
}

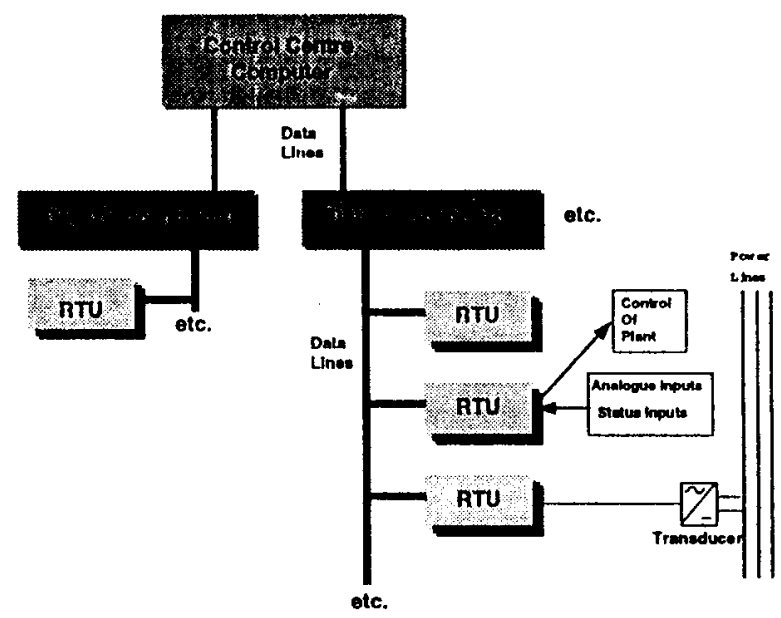

Figure 1. Network connections between control centre and power lines.

Whenever there is a fault, the analogues become unstable and read wrong values. 'This instability in analogue values is called "jitters". The jitters in analogues can be caused by several factors such as load fluctuations, faulty hardware and/or software in any part of the control equipment. If the jitter is due to load fluctuations, the analogue needs no analysis; otherwise, an analysis is needed.

Since the jitter analogues are not important when compared to other power system faults, such as system breakdown and power failure, they are always regarded as sccondary problems. As these analogues have some influence on the main power system faults, they cannot be neglected. To analyse the jittery analogues, an expert having relevant good experience and technical knowledge, is required. Since the expert is usually busy with other power system faults, it is always hard to find time to attend analogue problems. However, there is always a need for identification of the defective instruments which causes the jitters [2]. An alternate solution to alleviate the time pressure for the expert would be an on-line expert system. Since most of the analysis and diagnosis is based on the SCADA's data. there must be a direct interaction between SCADA and the expert system and, because of the direct data availability from SCADA, the system can be automated without any user interaction.

The approach described in this paper attempts to meet the industry's needs by analysing the jitter reporting analogs, finding the genuinely fault-related analogs and diagnosing 
them for the cause of jitter; and notifying the concerned technician about the problem. The characterisation of a knowledge based system for the above tasks in terms of knowledge, data and control of processing incorporated in the system is presented followed by a case study.

\section{ARCHITECTURE OF TPAISA}

The implementation of the expert system is on DEC VAX/VMS 3100 machine using IF/Prolog $V$ 4.1-8. It communicates with SCADA (on PDP-11/70) for accessing the necessary data required for the analysis and the diagnosis. The data thus acquired from SCADA are stored in 5 different databases and used at different instances of the problem solving process. The main problem solving knowledge of the domain expert is represented in rules which is termed "expert knowledge base" in this system. The general technical knowledge of the expert has been separated and included in another knowledge base called "general knowledge". Apart from these knowledge bases, the system incorporates another knowledge base termed "strategic common sense" which includes the common sense knowledge of the domain expert. The inference engine loops through the rules of the expert knowledge base, the general knowledge base, and uses the stratcgic common sense to analyse and diagnose the faults with the help of the data (expeit database) available from SCADA. Figure 2 shows the general architecture of this expert system.

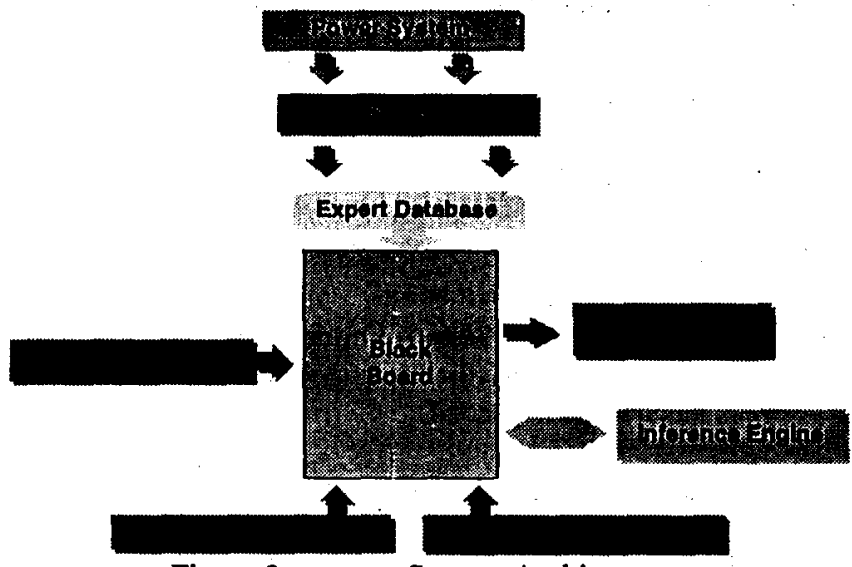

Figure 2.

System Architecture.

\section{EXPERT KNOWLEDGE AND DATA}

The knowledge included in this system is obtained from the control centre's sole expert in fault diagnosis. The process of the analysis starts with the identification of the analogs with jitter count which is a value indicating the number of times the analogue has been jittery within a specified length of timc. The maximum jitter count is called the "maximum' jitter record" and all the analogues iphich have the jitter count above $10 \%$ of this "maximum jitter record" are considered for further analysis. This preliminary exclusion eliminates the unimportant analogues from the 2080 analogues in the power system. The selected analogues are then divided into 5 groups: lake, rotary condenser, line, generator and transformer analogues. Lake and rotary condenser analogues are eliminated as their jitter is mainly due to genuine changes such as lake level changes and load fluctuations. The remaining analog groups (line, transformer; and generator) are further analysed by using procedures specific to each group. In general, when a jittery analogue is analysed, a clieck is made whether any other analogues from the same station or same RTU, are jittery. If they are jittery, then further analysis is carried by regarding all these analogues as a set. If this set not jittery, then the analogue is analysed as a single analogue. The analogue is then checked for its properties such as scanning priority $^{2}$ and fixed scalc deflection ${ }^{3}$ to find whether the analogue is operating under the normal conditions. Once the analogue's normality is established, the analysis moves to identify any parallel or series circuit analogs associated with the problematic analog. Then, the analog values of these analogs are compared. Depending on this comparison, further analysis is made which is highly heuristic in nature and involves several decision loops. For example, if the circuit containing the problem analogue has a parallel circuit, then the analog value is compared with that of the parallel circuit. If both values are similar, the process moves to the identification of several factors associated with the second analogue. During the process, attempts are made to establish the relationship between the two analogues with respect to the jitter and the problem that caused the jitter. During these attempts, the decision path leads to decision loops, and further the decision making process moves through the loops, closer it gets to the establishment of the fault. The output of this analysis suggests whether the jitter is fault-related, and also provides some indications of the possible location of the problem.

When the jitter is established to be fault related, the diagnosis is made depending on the initial information available from the analysis. Generally, there are 6 areas in the power system where there can be a possibility of a fault. These problem areas are:

- Transducers (Voltage and current).

- Analogue input ranging boards in RTUs.

- Other faults in RTU.

- Data Concentrators.

- Power System.

- SCADA.

The diagnosis involves choosing an appropriate fault area from the above areas and suggesting the technician of some possible faults in the problem area.

The knowledge process thus formatted, is then represented in the following three modules:

1. Expert Knowledge.

2. General Knowledge.

3. Common Sense.

\subsection{Expert Knowledge}

Expert knowledge is cmployed to:

- Analyse the jittery analogs.

\footnotetext{
2 Scanning priority is the priority assigned to every analogue in the. power system.

3 Fixed scale deflection is the maximum deflection rate assigned to each analogue. Whenever the analogue exceeds this rate of deflection, it sends a jitter signal to SCADA.
} 
- Determine whether the jitter is fault related.

- Suggest possible diagnosis.

The rules designed for the expert knowledge base contains the goal in the "action" part and the elements needed to satisfy the goal in the "conditions" part. Each goal is evaluated in a context which is the expert's functional frame work within which the analysis and diagnosis are carried out. If there cxists a condition related to "expert database" ,"general knowledge", or "common sense" among the conditions of a rule, a request is made by the inference engine to the respective data or knowledge base for its evaluation. Some example rules of this situation are shown below:.

\section{Expert-rule 1:}

IF the analogue's circuit has a parallel circuit ${ }^{4}$

THEN the analogue history of both the analogues must be compared.

\section{Expert-rule 2:}

IF two analogues are measured in series

ANDIF there is no power diversion between the two analogues

AND the ?MW5 of the analoguc and the ?MIV of the series analogue are same

TIIEN proceed to diagnosis.

Rule I needs establishment of the fact that the jittery analogue's circuit has a parallel circuit using the information from "general knowledge" on liow to find a parallel circuit and proceeds further with the analysis. In rule 2 , the inference needs the MW (Mega Watts) value from the expert system database to establish that both the values are identical. In general, it is seen that each condition in the rules based on "expert knowledge" has a procedure attached to it, which access the information necessary for their processing from the database and/or the other knowledge bases of the system.

\subsection{General Knowledge and Common Sense}

Domain experts in power system operations tend to use some heuristics which they regard as "general knowledge" 6 or "common sense". Most of these heuristics are commonly used for decision making and problem solving. The "general knowledge" involves basic technical knowledge such as ways to find a parallel circuit, a triangle rule, and ways to compare the analogues. The "common sensc" is basically the expert's strategic knowledge used in the diagnosis. A best cxamplc for this would be the choice of an appropriate fault area from the most probable range of problem areas.

3.2.1. Modelling General Knowledge: During the analysis of a problem, the expert uses general technical knowledge; for example, as a part of analysis, the liuman expert searches for a parallel or series line with respect to the line being analysed. The procedure for finding a series or parallel line is same for any circuit or power line. Knowlcuge like this can be

\footnotetext{
4 The underline section states that the condition has a procedure alfached to it.

5 The ? before a word indicates that it is a variable.

6 The general knowledge in this system is a combination of common sense and technical knowledge.
}

scparatcel from main expett knowvledge and sct in a scparalc knowledge base. This separation will reduce the confusion in inanagement of large knowledge bases and also improves the portability ${ }^{7}$ of the knowledge. Consider rule 1 stated in the previous section. To establish whether the circuit has a parallel circuit, the knowledge should have a procedure to find the parallel circuit. This is where the general knowledge is used. The knowledge of this knowledge base is also represented in IF... THIEN rules. The rule structure of the procedure to establish a parallel circuit would be as follows:

\section{Gencral rule 1:}

IF the analogue's line has a parallel line by observation

AND both the lines are connected to same bus THEN the analogue's circuit has a parallel circuit.

Note that the "conditions" part in the above rule involves some procedures. These procedures are again "general" in case and are included in the same knowledge base. Consequently, the rules in this knowledge base will not use the rules of any other knowledge base, and once the procedure is passed to this knowledge base, only the output is returned to the rulc which called for it. This is also onc of the reasons why this lnowledge is portable.

3.2.2. Modelling Strateric Common Scnse: During fault diagnosis, it is always pessible that there exists more than onc diagnostic solutions for a particular problem. In thesc situations, the decision is made to select the most opportunc diagnosis among the set of possible diagnoses. This decision making is done "unconsciously" by the human expert by analysing possible diagnoses against some predefined and preferred criteria; such as time and cost involved in the repair, as will be discussed later. This type of decision making involves strategic common sense which can be modelled in an expert system using a method proposed by Mussi [1]. The implementation of our system is based on this method.

In this implementation, each diagnosis will have a certain belief level with respect to the presence of the fault (eg. probable, most_probablc, certain). This belief level states the extent of the surety in the diagnostic result. Let us take the following diagnostic rule from the expert knowledge base:

initial_diag_rule 1:

IF only one ?analogue of ?RTU is jitlery

THEN the presence of problem in ?word or ?card of ?RTU is most_probable.

This rule states the fact that if only one analogue of the RTU is jittery, then it is most probable that the "word address" or the "card address" of timat RTU is faulty. At this stagc, the information available from the analysis, may also point to possible problems in SCADA (wrong inputs, communication problems, etc.), and it cannot be ruled out. To makc thic presence of the problem morc "certain", further investigation has to be done by applying a special set of rules which always provide an output depending on the truth value of its

\footnotetext{
7 The general knowledge used in this system can be used in other
} power system fault diagnostic systems. 
conditions. These outputs are in the form of evidences with a certainty factor associated with it, and are used in the final diagnostic rules which are the final decision making rules having the "evidences" in their "conditions" part and the related diagnosis in the "conclusions" part of the rule. If all the "conditions" of a rule are true, then the diagnosis will have the highest value of the belief level. This diagnosis (with highest "value") is chosen as the final diagnostic solution. Contradiction arises when more_than_one rule satisfies all their "conditions" (multiple final solutions) or no rule satisfies all the "conditions" (no final solution). It is here that the "strategic common sense" is used to resolve the contradictions.

3.2.3. Strategic Common Sense: Apart from the belief level, each diagnosis has some "preference parameters" (such as evidences, time_consuming, and cost) attached to it. Each preference parameter has a range of values: low, medium, high. The selection of diagnosis using "strategic common sense" is based on the "preference criteria" which refer to preference parameters and their values. For example, if $\mathbf{P}$ is the preference parameter "evidence", a likely "preference criterion" would be: "select the diagnosis with highest evidences". There are similar preference criteria for the other preference parameters.

A diagnostic solution which satisfies all the "preference criteria" is chosen as the most opportune solution. However, it is hard to find a diagnostic solution which has

evidence $\gg>$ high;

time_consuming $\gg>$ low;

cost $\gg$ > low;

and so on.

Some diagnoses might have "high" evidence but it may be "more" time consuming to solve or involve "high" cost. Hence there is a need to select the diagnosis by prioritising the preference criteria. This priority has to be defined by human experts. During human expert's diagnosis, this prioritisation is "unconsciously" done. This "unconscious" knowledge can be derived from human expert's rules by analysing them [1]. This meta-knowledge base is represented as meta-rules which act as strategic common sense. An example of a meta-rule is given below for two preference criteria $X$ and $Y$, where $X=$ "Select the diagnosis with lowest value of time_consuming", and $\mathrm{Y}=$ "Select the diagnosis with lowest cost value", and $\mathrm{X}$ is more important than $\mathrm{Y}$ by domain expert's initial priority assignment:

common_sense_r_1:

IF Fin_diag_1_pparvX = Fin_diag_2_pparvX

THEN choose between Fin_diag_1 and Fin_diag_2 with respect to $Y$

where, Fin_diag_1 and Fin_diag_2 are the two conflicting diagnostic rules and pparvX is the value of the preference parameter of the preference criterion $X$.

The above rule states that, if the preference parameter value of the preference criterion $X$ (eg. time_consuming of diagnosis_l $\gg$ >nedium) of one of the conflicting diagnosis is equal to that of the other diagnosis (eg. time_consuming of diagnosis_2 $\gg$ medium) then select the best diagnosis with respect to the value of preference criterion $Y$ (ie. $\cos t$ ).
The above mentioned tule is the simplest meta-rule in this knowledge base and is applicable if there are only two conflicling diagnoses and each one of those has only two preference criteria attached to them. More Complex rules are usted when there are more than two diagnostic rules in conflict and each rule has more than two preference criteria. A more detailed description of these rules and strategic common sense can be found in Rayudu [3].

\section{EXPERT DATABASE}

To integrate an on-line system with SCADA, the real time network data has to be stored in a database in such a way that they can be accessed by the system. Such databases are already in use by other application programs in the control centre but they do not provide all the information our system needs. Hence we had to develop our own database. The database stores the information in five different levels developed in object oriented representational approach (Figure 3). Level 1 \& 2 stores the information of jitter analogues and their values. Level 3 consists of the RTU information and the analogues attached to each of them. The data concentrator information are stored in level 4 along with RTUs addresses connected to it and the final level consists of the power system network data necessary for the expert system. In many situations, the access of the database is fairly hierarchical with respect to the stages of the analysis and diagnosis.

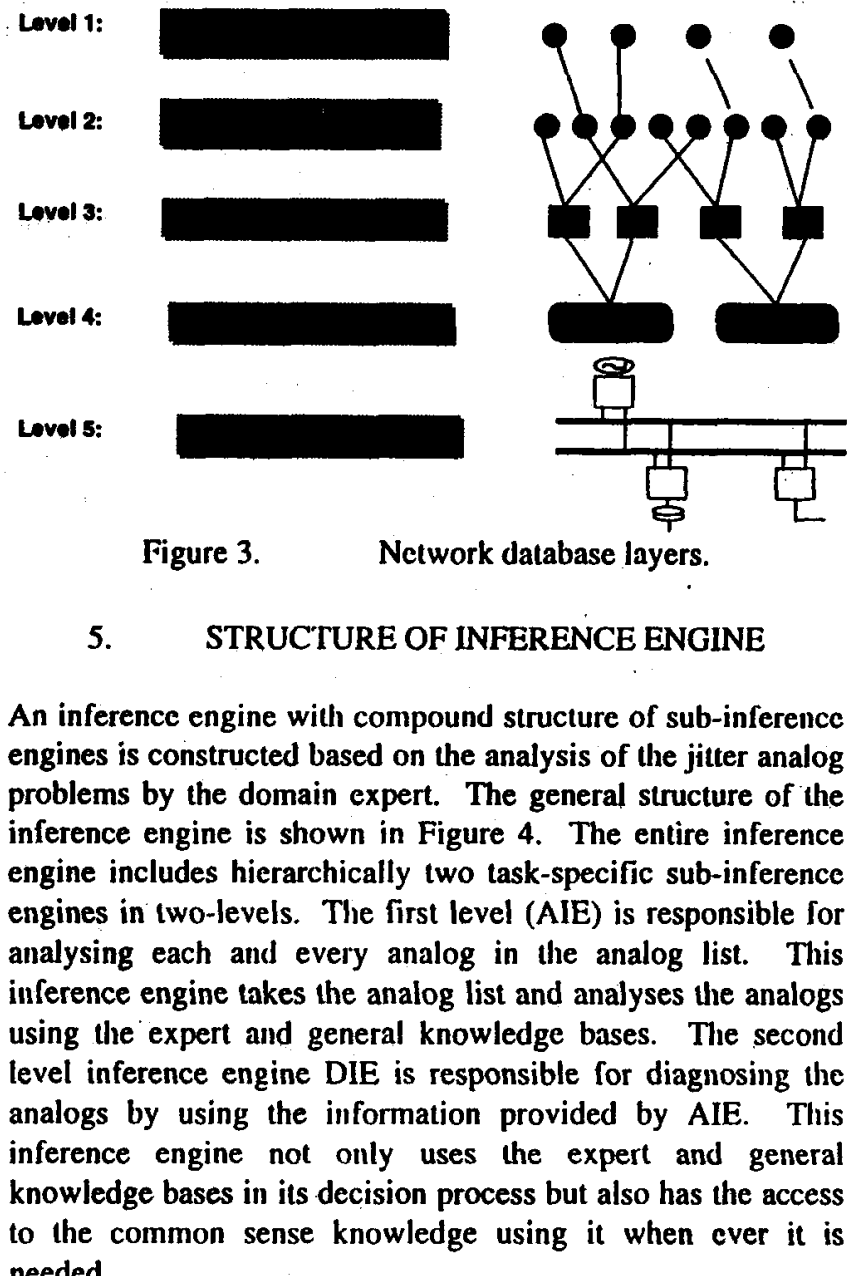
needed. 


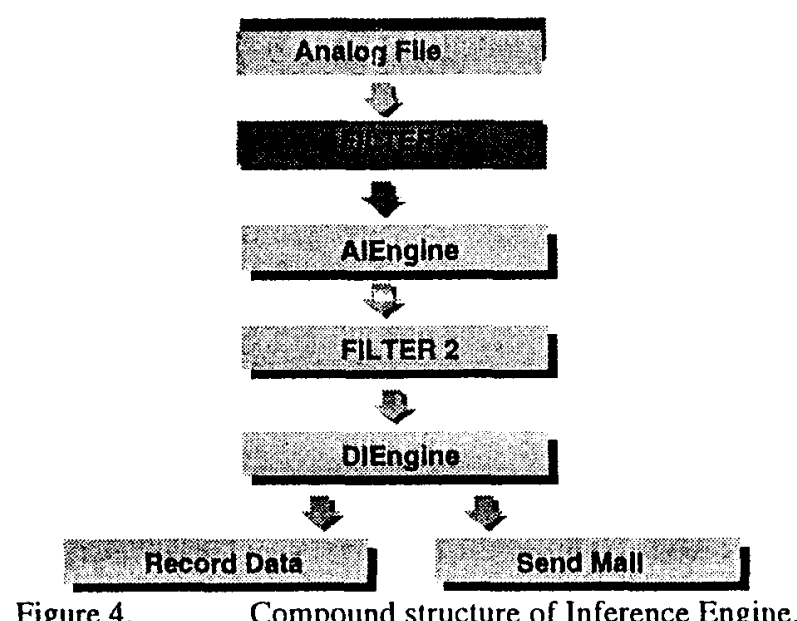

Figure 4. Compound structure of Inference Engine.

TPAISA's inference engine uses a combination of backward chaining and forward chaining mechanisms and some logical control structures from traditional programming. In the expert system, backward chaining is used in the "fault analysis", and forward chaining is used in the "fault diagnosis". Control structures are used in firing of "final fault diagnosis" rules where all the rules succeed whether or not all the "conditions" of each rule are "true". The control structures of standard programming are also used in searching.

The interface between the expert system and SCADA is done in $R T L / 2$ language. This interface transports the necessary real-time information from SCADA to the "expert database" where it is stored in the levels specified.

The entire system is designed to operate "automatically" without any user interaction and only at a specific time it shuts itself off after the diagnosis. The output of the operation is presented in the form of a list of "reports" usually sent as a mail message to the concerned technical personnel. Other facilities, such as keeping track of the most jittery analogues and keeping a record of problem frequency of each analogue are also provided. In addition, a database of diagnosed analogs is maintained.

\section{CASE STUDY}

A part of jitter analog data obtained from SCADA is shown in the Figure 5.

$\begin{array}{ll}\text { ANALOG } & \text { Jitter Count } \\ & \\ \text { WPRASYMW } & 543 \\ \text { WPRASYMV } & 213 \\ \text { SBKKAIMW } & 32 \\ \text { TMKT2TAB } & 108 \\ \text { UTKCOB2A } & 89 \\ \text { ROXT1NiV } & 57 \\ \text { AVIBEN1A } & 446 \\ \text { AVIBEN1MV } & 0 \\ \text { BRYASBA } & 86 \\ \text { INVGORA } & 146 \\ \text { INVGORMW } & 103 \\ \text { INVGORV } & 132 \\ \text { WPRASYA } & 36 \\ \text { TMKT4MW } & 0 \\ \text { BENG6MW } & 12\end{array}$

Figure 5. Sample Analog list with jitter counts.
This file is then fed to TPAISA. The preliminary filter (FILTER 1 in Fig. 4) filters all the unnecessary analogs and the resulting analog list is sent for analysis. The analysis of these analogs is carried by Analysis Inference Engine (AIE) where every analog is analysed. After the analysis is complete, a second filter is used to filter the analogs based on the output from AIE. The output list from FILTER 2 is then used for diagnosis of the analogs. The diagnosis is done by Diagnosis Inference Engine (DIE) where the output result is then written onto two files: one for sending to the technician an another for records. An Example of the diagnostic output of TPAISA is shown in the Figure 6.

$\begin{array}{llll}\text { ANALOG } & \text { RTU } & \text { CA } & \text { WA } \\ \begin{array}{l}\text { WPRASYMW } \\ \text { WPRASYMV }\end{array} & 2 & 3 & 6 \\ & 2 & 3 & 4 \\ \text { Diagnosis: Faulty card 104. } & & & \\ & & & \\ \text { AVIBEN1A } & 6 & 2 & 8 \\ \text { Diagnosis: Check Card 104. } & & & \\ \begin{array}{l}\text { INVGORA } \\ \text { INVGORMW }\end{array} & 5 & 3 & 3 \\ \text { INVGORV } & 5 & 4 & 2 \\ \end{array}$

Diagnosis: Check the transducers.

Figure 6. Sample of diagnosed output from TPAISA.

The experience with a number of TPAISA has shown that:

1. In general, problem analogs are identified and are sent to the technician without any user interaction, thereby saving the expert's time.

2. The procedure presented here provides satisfactory results for larger extent of the jitter problems. Seventy percent of the analysed analog list were found to be accurate (i.e. analogs which jitter due to faults). This is closer to our expectations as the knowledge encoded in TPAISA, according to the domain expert, is capable of solving only $84 \%$ of the jitter analog problems.

\section{CONCLUSION}

A power system fault analysis and diagnosis expert system is developed with the incorporation of "general knowledge" and "strategic common sense" as decision tools in conjunction with the domain expert's problem solving knowledge. The system is an "automatic" on-line operating software which runs once a day to analyse and diagnose the "jitter" analogues of the power system. The expert knowledge base consists of the domain expert's heuristic rules which basically drive the inferences of the system. The general knowledge base consists of rules relating to the general technical knowledge of the power systems. It is used where there is a need for general observations in the problem solving process. The common sense knowledge base consists of meta-rules relating to the unconscious decision making strategy of the domain expert. This knowledge is used in the problem diagnosis part where the conflicting diagnostic rules are resolved for the most promising diagnostic solution. 
The separation of expert and general knowledge improves the maintainability of the knowledge bases and also helps in knowledge portability and reuse. Research is underway to investigate the portability and reuse of these knowledge bases as the expert system will be applied to the other control centrc ${ }^{8}$ in the future.

\section{ACKNOWLEDGEMENTS}

The authors would like to thank all the staff and technicians at the control centre, particularly, Mr. Tony Silke, Mr. Ken Papps, Mr. Adan Thomas, Mr. David Peters and Mr. Kevin Williams for their help and support towards this research.

Our special thanks goes to the only domain expert of this project, Mr. Les Jones, Supervising Technician, Power Mark Ltd.

\section{REFERENCES}

[1] Silvano Mussi, "A Method for putting stratcgic common sense into expert systems", IEEE Trans. on Knowledge and Data Engineering, Vol. 5, No. 3, June 1993, pp. 369-385.

[2] Adibi M. M., Kafka R. J., Clements K. A., and Stovall J. P., "Integration of Remote Measurement Calibration with State Estimation - A Feasibility Study", IEEE Trans. on Power Systèms, vol. 7, no. 3, pp. 1164-1172, Aug. 1992.

[3] Rayudu R. K., Ken P., Tony S., and Sandhya S., "Towards the design of an Intelligent System in Power Systems", Proc. 10th CEPSI, Christchurch, New Zealand, September, 1994, pp. 310-320.

[4] Sugihara H., "A Practical Expert System for indicating faulty sections within a control centre", ESAP' 1993 , Australia, pp. 236-241.

[5] Monika P., and Nejdl W., "Intcgrating Model based and Heuristic Features in a Real-Time Expert System", IEEE Expert, August, 1993, pp. 1L-18.

[6] Adibi M. M. and Kalka R. J., "Minimization of Uncertainties in Analog Measurements for use in State Estimation", IEEE Trans. on Power Systems, vol. 5, no. 3, pp. 902-910, Aug. 1990.

8 The control centre referred to, is the North Island Control Centre; the control centre which controls the North Island's power transmission in New Zealand.. 